\title{
Glycogen Synthase Kinase 3: A Point of Integration in Alzheimer's Disease and a Therapeutic Target?
}

\author{
Siddhartha Mondragón-Rodríguez, ${ }^{1}$ George Perry, ${ }^{2,3}$ Xiongwei Zhu, ${ }^{3}$ \\ Paula I. Moreira, ${ }^{4,5}$ and Sylvain Williams ${ }^{1}$ \\ ${ }^{1}$ Department of Psychiatry, Douglas Hospital Research Center, McGill University, Montreal, QC, Canada H4H 1R3 \\ ${ }^{2}$ UTSA Neurosciences Institute and Department of Biology, College of Sciences, The University of Texas at San Antonio, \\ San Antonio, TX, USA \\ ${ }^{3}$ Department of Pathology, Case Western Reserve University, Cleveland, OH, USA \\ ${ }^{4}$ Center for Neuroscience and Cell Biology, University of Coimbra, Coimbra, Portugal \\ ${ }^{5}$ Faculty of Medicine, Institute of Physiology, University of Coimbra, Coimbra, Portugal
}

Correspondence should be addressed to Siddhartha Mondragón-Rodríguez, sidmonrod@gmail.com

Received 7 February 2012; Revised 20 April 2012; Accepted 3 May 2012

Academic Editor: Francesco Panza

Copyright () 2012 Siddhartha Mondragón-Rodríguez et al. This is an open access article distributed under the Creative Commons Attribution License, which permits unrestricted use, distribution, and reproduction in any medium, provided the original work is properly cited.

\begin{abstract}
Glycogen synthase kinase 3 (GSK3) has been implicated in neurological disorders; therefore, it is not surprising that there has been an increased focus towards developing therapies directed to this kinase. Unfortunately, these current therapies have not taken into consideration the physiological role of GSK3 in crucial events like synaptic plasticity. With this in mind we will discuss the relationship of synaptic plasticity with GSK3 and tau protein and their role as potential targets for the development of therapeutic strategies. Finally, we will provide perspectives in developing a cocktail therapy for Alzheimer's treatment.
\end{abstract}

\section{Introduction}

Glycogen synthase kinase 3 (GSK3) is an evolutionarily conserved protein that is active in resting cells and is inhibited in response to activation of several distinct pathways such as the Wnt, insulin, and the growth factor pathway [1-7]. GSK3 activity is regulated by different mechanisms, including (a) phosphorylation at an $\mathrm{N}$-terminal serine $[7,8]$, (b) through phosphorylation of a tyrosine residue [9], (c) through phosphorylation of a C-terminal serine residue [10], and (d) through disruption of the axin- $\beta$-catenin multiprotein complex $[4,11,12]$. The other requirement of GSK3 is that most of its substrates require prior phosphorylation at residue 4 or 5 amino acids C-terminal to the target residue [13].

GSK3 has two isoforms GSK3 $\alpha$ and GSK3 $\beta$, which are encoded by different genes [14]. In mouse, rat, and human, an alternative isoform (GSK3 $\beta 2$ ) that contains a 13-aminoacid insert near the catalytic domain was reported [15]. In opposition to GSK $3 \alpha$ and GSK3 $\beta$, GSK3 $\beta 2$ is specifically found in the nervous system and has been strongly linked to neurodevelopment [15].

In order to participate in all these events, GSK3 has a broad range of substrates: cyclic AMP response elementbinding protein (CREB), neurogenin 2, SMAD1, NFkappaB, Myc, heat shock factor-1, cyclin D1, nuclear factor of activated T-cells and CCAAT/enhancer-binding proteins, cJun, $\beta$-catenin, and microtubule-associated proteins like MAP2 and tau [16-18]. GSK3 regulates some of these factors by controlling their protein levels. However, changes in GSK3 activity have been associated with neurodegenerative diseases, such as bipolar disorder, schizophrenia, and Alzheimer's disease (AD) [19]. Indeed, in AD the active form of GSK $3 \beta$ was found to be directly related to the hyperphosphorylation of tau present in paired helical filament (PHF)tau of neurofibrillary tangles (NFTs) [20]. Importantly and due to the fact that most drugs bind and compete with ATP, there appears to be only a single amino acid difference (Glu196 in GSK3 $\alpha$, Asp133 in GSK3 $\beta$ ) making it difficult to identify an inhibitor that can distinguish the two isoforms. 
Overall, it is clear that GSK3 is related to AD development, and, more importantly, current data suggest that both isoforms (GSK3 $\alpha$ and GSK3 $\beta$ ) contribute to $\mathrm{AD}$ pathogenesis.

\section{Tau Pathology and GSK3}

Tau is an axonal protein that regulates microtubule stability [21]; however, during $\mathrm{AD}$ tau is abnormally phosphorylated and aggregates into NFTs [22, 23]. Tau has at least 45 phosphorylation sites, mostly located in the prolinerich region (P-region) (residues 172-251) and the Cterminal tail region (C-region) (residues 368-441) [24]. Tau phosphorylation at both of these regions affects its capacity to interact with microtubules [25]. In terms of $\mathrm{AD}$ pathology, the phosphorylation sites located in the Cterminal region seem to cause (a) abnormal folding and (b) protein cleavage, which together could lead to tau deposition [26-28]. Phosphorylation at some sites (Ser262) selectively impairs binding of tau to microtubules [29], whereas phosphorylation at other sites (Ser202) enhances tau polymerization [30]. Crucially, GSK3 $\beta$ has been linked to many of these sites $[15,31]$. Therefore, emphasis has been placed particularly on GSK $3 \beta$, rather than GSK $3 \alpha$. However, due to the lack of inhibitor's specificity, GSK $3 \alpha$ has not been ruled out. Indeed, some studies have shown that GSK $3 \alpha$ through Wnt signalling pathway is also related to tau pathology [32]. Furthermore, by specifically knocking down GSK $3 \beta, \operatorname{GSK} 3 \alpha$ was found to be related to AD pathology [33].

In sum, the current data shows that both isoforms GSK3 $\alpha$ and GSK $3 \beta$ could be involved in tau phosphorylation.

\section{GSK3 as the Therapeutic Target for AD}

GSK3 is strongly implicated in neurodegeneration [34], and, not surprisingly, it has been postulated as a therapeutic target in the treatment of $\mathrm{AD}$. Indeed, lithium which is a direct inhibitor of both GSK $3 \beta$ and GSK $3 \alpha$ has been used in humans $[35,36]$. The direct regulation of GSK3 also modifies cell survival as it is known for facilitating a variety of apoptotic mechanisms [35]. Similarly, in an attempt to reduce tau pathology, the GSK3 inhibitor [Tideglusib/NP-12 (Nypta)] is currently in clinical trial [37]. NP-12 has been designated as an orphan drug by the EU and US authorities and has been granted Fast Track status by the FDA (see http://www.noscira.com).

The rationale is simple; blocking GSK3 will lead to nonphosphorylated tau and, consequently, less tau deposition according to the current hypothesis. However, the importance of GSK3 for normal physiological cell functioning must be taken into consideration. In this regard, we recently found that phosphorylation of tau protein is critical in order for the protein to function as a negative feedback mechanism to prevent NMDA-receptor overexcitation (unpublished data). This data becomes crucial in this debate since NMDA deregulation plays a vital role in synaptic plasticity. Therefore, by simple blockade of GSK3 we could alter the homeostasis of synaptic plasticity among other important physiological functions. Furthermore, blocking GSK3 also raises the possibility of affecting gene expression and cell survival [17]. So, is GKS3 the desired therapeutic target for AD? Although the answer is far from being simplistic, normal physiological functions for the cell, together with the complexity of the phenomena [38], need to be taken into consideration before selecting AD pharmacological targets.

\section{GSK3 as Crucial Node for Synaptic Plasticity}

Synaptic plasticity has been proposed to play a central role in brain capacity to incorporate transient experiences into persistent memory traces. Synaptic transmission can be enhanced (long-term potentiation, LTP) or depressed (long-term depression, LTD) by activity, and these changes can persist from seconds to hours and days [39, 40]. Importantly, the affected intracellular pathways leading to LTP or LTD activation involve primarily GSK3 [41, 42]. Indeed, it has been shown that enhanced GSK3 signalling impairs hippocampal memory formation [43]. Specifically, GSK3 activity blocks synaptic LTP and induces LTD [43]. Furthermore, it was found that GSK3 during LTP involves activation of NMDA receptors and the PI3K-Akt pathway consequently disrupting the ability of synapses to undergo LTD [43]. Clearly, the data claims that GSK3 is a crucial node mediating the LTP to LTD transition. Therefore, the simple idea of blocking GSK3 in order to prevent the progression of AD seems to be overly simplistic.

\section{Conclusion and Perspectives}

The hypothesis that GSK3 plays a role in the aetiology of brain disorders is further nurtured by the fact that several genetic susceptibility factors for psychiatric disorders have key roles in neurodevelopment. Importantly, many of the genes are involved in GSK3 signaling [44, 45]. Furthermore, GSK3 is directly related to the pathogenesis of AD as tau kinase [31]. Overall, it seems clear that GSK3 has an integral role in the pathogenesis of AD. Therefore, GSK3 remains as therapeutic target. However, the secondary effects caused by GSK3 blockade should also be taken into consideration, especially knowing that synaptic dysfunction in addition to neuronal death can lead to cognitive failure associated with AD. With this in mind, therapies that focus on rescuing events like LTP rather than single blocking strategy could bring needed results.

In conclusion, we suggest that downstream targets of GSK3 are an interesting option. In other words, we proposed the use of cocktail drugs that could enhance LTP and reduce induction of LTD. For instance, drugs like memantine (NMDA receptor antagonist) [46], in combination with other drugs like okadaic acid (PP1 activator) [47] and/or pseudosubstrate for Akt [43], could be used in order to balance the activity of GSK3 and therefore tau phosphorylation (Figure 1). Together, this combinatorial approach may result in LTP promotion and synaptic improvement. After all, if the current strategies for $\mathrm{AD}$ treatment have shown 


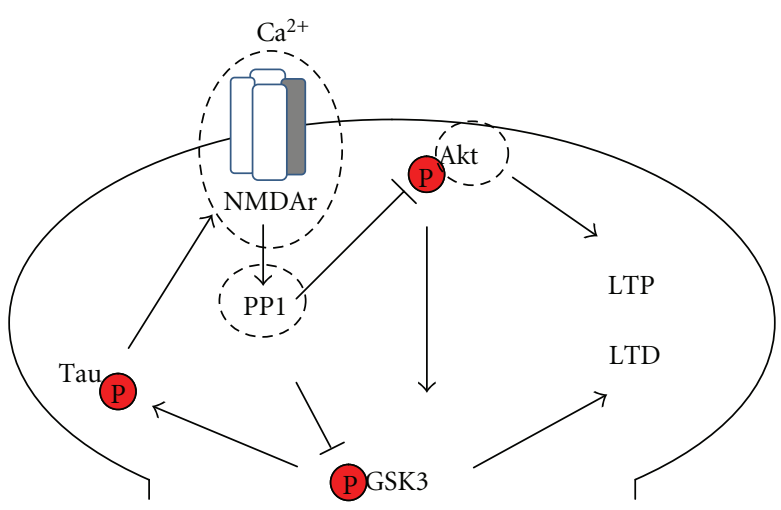

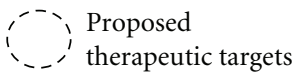

FIGURE 1: Cocktail drugs could balance the activity of GSK3 during AD. The role of PP1 and Akt in GSK3 activation, in combination with NMDA receptor, makes them important therapeutic targets. Calcium $\left(\mathrm{Ca}^{2+}\right)$ enters via NMDA receptors, and this leads to activation of protein phosphatase 1 (PP1), a key enzyme in synaptically induced LTD. PP1 can dephosphorylate GSK3 that determines whether NMDA receptor activation induces LTD or inhibits LTD. PP1 can dephosphorylate Akt, resulting in GSK3 activation. GSK3, under the control of Akt and PP1, is a critical determinant of the direction of NMDA receptor-dependent plasticity. The active GSK3 isoforms contribute to phosphorylation of tau protein which is essential in order for the protein to function as a negative feedback mechanism to prevent NMDA-receptor overexcitation and synaptic failure.

little benefits, it is tempting to consider new therapeutic approaches that are aimed to improve memory formation.

\section{Abbreviations}

AD: Alzheimer's disease

GSK3: Glycogen synthase kinase 3

NFTs: Neurofibrillary tangles

LTP: Long-term potentiation

LTD: Long-term depression

NMDA: N-methyl-D-aspartate receptor.

\section{Disclosure}

George Perry is, or has in the past been, a paid consultant for and/or owns equity or stock options in Neurotez Pharmaceuticals, Panacea Pharmaceuticals, Takeda Pharmaceuticals, and Voyager Pharmaceuticals. The authors have no other relevant affiliations or financial involvement with any organization or entity with a financial interest in or financial conflict with the subject matter or materials discussed in the paper apart from those disclosed.

\section{Acknowledgments}

The authors thank Jesse Jackson for critical comments. Work in the authors' laboratories is supported by Conacyt, Mexico;
Alzheimer's Association, USA; Alzheimer Society, Canada; CIHR, Canada and FRSQ, Québec, Canada. This project was supported by grants from the National Center for Research Resources (5 G12RR013646-12), the National Institute on Minority Health and Health Disparities (G12MD007591) from the National Institutes of Health, and from the Research Centers in Minority Institutions (RCMI).

\section{References}

[1] D. Wu and W. Pan, "GSK3: a multifaceted kinase in Wnt signaling," Trends in Biochemical Sciences, vol. 35, no. 3, pp. 161-168, 2010.

[2] C. Metcalfe and M. Bienz, "Inhibition of GSK3 by Wnt signalling - two contrasting models," Journal of Cell Science, vol. 124, no. 21, pp. 3537-3544, 2011.

[3] V. F. Taelman, R. Dobrowolski, J. L. Plouhinec et al., "Wnt signaling requires sequestration of glycogen synthase kinase 3 inside multivesicular endosomes," Cell, vol. 143, no. 7, pp. 1136-1148, 2010.

[4] J. Papkoff and M. Aikawa, "WNT-1 and HGF regulate GSK3 $\beta$ activity and $\beta$-catenin signaling in mammary epithelial cells," Biochemical and Biophysical Research Communications, vol. 247, no. 3, pp. 851-858, 1998.

[5] Y. Saito, J. R. Vandenheede, and P. Cohen, "The mechanism by which epidermal growth factor inhibits glycogen synthase kinase 3 in A431 cells," Biochemical Journal, vol. 303, no. 1, pp. 27-31, 1994.

[6] D. A. E. Cross, D. R. Alessi, J. R. Vandenheede, H. E. McDowell, H. S. Hundal, and P. Cohen, "The inhibition of glycogen synthase kinase- 3 by insulin or insulin-like growth factor 1 in the rat skeletal muscle cell line L6 is blocked by wortmannin, but not by rapamycin: evidence that wortmannin blocks activation of the mitogen-activated protein kinase pathway in L6 cells between Ras and Raf," Biochemical Journal, vol. 303, no. 1, pp. 21-26, 1994.

[7] C. Sutherland, I. A. Leighton, and P. Cohen, "Inactivation of glycogen synthase kinase- $3 \beta$ by phosphorylation: new kinase connections in insulin and growth-factor signalling," Biochemical Journal, vol. 296, no. 1, pp. 15-19, 1993.

[8] V. Stambolic and J. R. Woodgett, "Mitogen inactivation of glycogen synthase kinase- $3 \beta$ in intact cells via serine 9 phosphorylation," Biochemical Journal, vol. 303, no. 3, pp. 701-704, 1994.

[9] P. A. Lochhead, R. Kinstrie, G. Sibbet, T. Rawjee, N. Morrice, and V. Cleghone, "A chaperone-dependent GSK3 $\beta$ transitional intermediate mediates activation-loop autophosphorylation," Molecular Cell, vol. 24, no. 4, pp. 627-633, 2006.

[10] T. M. Thornton, G. Pedraza-Alva, B. Deng et al., "Phosphorylation by 38 MAPK as an alternative pathway for GSK3 $\beta$ inactivation," Science, vol. 320, no. 5876, pp. 667-670, 2008.

[11] G. Wu, H. Huang, J. G. Abreu, and X. He, "Inhibition of GSK3 phosphorylation of $\beta$-catenin via phosphorylated PPPSPXS motifs of Wnt coreceptor LRP6," PLoS ONE, vol. 4, no. 3, Article ID e4926, 2009.

[12] K. M. Cadigan and Y. I. Liu, "Wnt signaling: complexity at the surface," Journal of Cell Science, vol. 119, no. 3, pp. 395-402, 2006.

[13] S. Frame, P. Cohen, and R. M. Biondi, "A common phosphate binding site explains the unique substrate specificity of GSK3 and its inactivation by phosphorylation," Molecular Cell, vol. 7, no. 6, pp. 1321-1327, 2001. 
[14] J. R. Woodgett, "Molecular cloning and expression of glycogen synthase kinase-3/factor A," The EMBO Journal, vol. 9, no. 8, pp. 2431-2438, 1990.

[15] F. Mukai, K. Ishiguro, Y. Sano, and S. C. Fujita, "Aternative splicing isoform of tau protein kinase I/glycogen synthase kinase $3 \beta$," Journal of Neurochemistry, vol. 81, no. 5, pp. 10731083, 2002.

[16] F. Al-Mulla, M. S. Bitar, M. Al-Maghrebi et al., "Raf kinase inhibitor protein RKIP enhances signaling by glycogen synthase kinase-3 $\beta$," Cancer Research, vol. 71, no. 4, pp. 13341343, 2011.

[17] C. A. Grimes and R. S. Jope, "The multifaceted roles of glycogen synthase kinase $3 \beta$ in cellular signaling," Progress in Neurobiology, vol. 65, no. 4, pp. 391-426, 2001.

[18] M. P. M. Soutar, W. Y. Kim, R. Williamson et al., "Evidence that glycogen synthase kinase-3 isoforms have distinct substrate preference in the brain," Journal of Neurochemistry, vol. 115, no. 4, pp. 974-983, 2010.

[19] R. S. Jope and M. S. Roh, "Glycogen synthase kinase-3 (GSK3) in psychiatric disease and therapeutic interventions," Current Drug Targets, vol. 7, no. 11, pp. 1421-1434, 2006.

[20] K. Leroy, A. Boutajangout, M. Authelet, J. R. Woodgett, B. H. Anderton, and J. P. Brion, "The active form of glycogen synthase kinase- $3 \beta$ is associated with granulovacuolar degeneration in neurons in Alzheimers's disease," Acta Neuropathologica, vol. 103, no. 2, pp. 91-99, 2002.

[21] G. V. W. Johnson and J. A. Hartigan, "Tau protein in normal and Alzheimer's disease brain: an update," Journal of Alzheimer's Disease, vol. 1, no. 4-5, pp. 329-351, 1999.

[22] G. Farias, A. Cornejo, J. Jiménez, L. Guzmán, and R. B. Maccioni, "Mechanisms of tau self-aggregation and neurotoxicity," Current Alzheimer Research, vol. 8, no. 6, pp. 608-614, 2011.

[23] G. V. W. Johnson and W. H. Stoothoff, "Tau phosphorylation in neuronal cell function and dysfunction," Journal of Cell Science, vol. 117, no. 24, pp. 5721-5729, 2004.

[24] D. P. Hanger, B. H. Anderton, and W. Noble, "Tau phosphorylation: the therapeutic challenge for neurodegenerative disease," Trends in Molecular Medicine, vol. 15, no. 3, pp. 112119, 2009.

[25] F. Liu, B. Li, E. J. Tung, I. Grundke-Iqbal, K. Iqbal, and C. X. Gong, "Site-specific effects of tau phosphorylation on its microtubule assembly activity and self-aggregation," European Journal of Neuroscience, vol. 26, no. 12, pp. 3429-3436, 2007.

[26] S. Mondragon-Rodriguez B.-I. G. and F. García-Sierra, "The chronology and maturation of the neurofibrillary tangle pathology in Alzheimer's disease is based on the state of phosphorylation, conformational changes, and cleavage of tau protein," Novascience Book. In press.

[27] S. Mondragón-Rodríguez, G. Basurto-Islas, I. Santa-Maria et al., "Cleavage and conformational changes of tau protein follow phosphorylation during Alzheimer's disease," International Journal of Experimental Pathology, vol. 89, no. 2, pp. 8190, 2008.

[28] S. Mondragón-Rodríguez, G. Basurto-Islas, L. I. Binder, and F. García-Sierra, "Conformational changes and cleavage; are these responsible for the tau aggregation in Alzheimer's disease?" Future Neurology, vol. 4, no. 1, pp. 39-53, 2009.

[29] D. Fischer, M. D. Mukrasch, J. Biernat et al., "Conformational changes specific for pseudophosphorylation at serine 262 selectively impair binding of tau to microtubules," Biochemistry, vol. 48, no. 42, pp. 10047-10055, 2009.

[30] C. A. Rankin, Q. Sun, and T. C. Gamblin, "Pseudophosphorylation of tau at Ser202 and Thr205 affects tau filament formation," Molecular Brain Research, vol. 138, no. 1, pp. 84-93, 2005.

[31] B. A. J. Schaffer, L. Bertram, B. L. Miller et al., "Association of GSK3B with Alzheimer disease and frontotemporal dementia," Archives of Neurology, vol. 65, no. 10, pp. 1368-1374, 2008.

[32] A. A. Asuni, C. Hooper, C. H. Reynolds, S. Lovestone, B. H. Anderton, and R. Killick, "GSK $3 \alpha$ exhibits $\beta$-catenin and tau directed kinase activities that are modulated by Wnt," European Journal of Neuroscience, vol. 24, no. 12, pp. 33873392, 2006.

[33] C. J. Phiel, C. A. Wilson, V. M. Y. Lee, and P. S. Klein, "GSK-3 $\alpha$ regulates production of Alzheimer's disease amyloid$\beta$ peptides," Nature, vol. 423, no. 6938, pp. 435-439, 2003.

[34] M. D. Kaytor and H. T. Orr, "The GSK $3 \beta$ signaling cascade and neurodegenerative disease," Current Opinion in Neurobiology, vol. 12, no. 3, pp. 275-278, 2002.

[35] R. S. Jope and G. N. Bijur, "Mood stabilizers, glycogen synthase kinase- $3 \beta$ and cell survival," Molecular Psychiatry, vol. 7, supplement 1, pp. S35-S45, 2002.

[36] C. T. Chiu and D. M. Chuang, "Neuroprotective action of lithium in disorders of the central nervous system," Zhong Nan Da Xue Xue Bao Yi Xue Ban, vol. 36, no. 6, pp. 461-476, 2011.

[37] L. Gravitz, "Drugs: a tangled web of targets," Nature, vol. 475, no. 7355, pp. S9-S11, 2011.

[38] S. Mondragón-Rodríguez, G. Basurto-Islas, H.-G. Lee et al., "Causes versus effects: the increasing complexities of Alzheimer's disease pathogenesis," Expert Review of Neurotherapeutics, vol. 10, no. 5, pp. 683-691, 2010.

[39] J. G. Howland and Y. T. Wang, "Synaptic plasticity in learning and memory: stress effects in the hippocampus," Progress in Brain Research, vol. 169, pp. 145-158, 2008.

[40] V. M. Ho, J.-A. Lee, and K. C. Martin, "The cell biology of synaptic plasticity," Science, vol. 334, no. 6056, pp. 623-628, 2011.

[41] G. L. Collingridge, S. Peineau, J. G. Howland, and Y. T. Wang, "Long-term depression in the CNS," Nature Reviews Neuroscience, vol. 11, no. 7, pp. 459-473, 2010.

[42] V. J. Appleby, S. A. L. Corrêa, J. K. Duckworth et al., "LTP in hippocampal neurons is associated with a CaMKIImediated increase in GluA1 surface expression," Journal of Neurochemistry, vol. 116, no. 4, pp. 530-543, 2011.

[43] S. Peineau, C. Taghibiglou, C. Bradley et al., "LTP inhibits LTD in the hippocampus via regulation of GSK3beta," Neuron, vol. 53, no. 5, pp. 703-717, 2007.

[44] J. B. Kwok, C. T. Loy, G. Hamilton et al., "Glycogen synthase kinase-3beta and tau genes interact in Alzheimer's disease," Annals of Neurology, vol. 64, no. 4, pp. 446-454, 2008.

[45] N. Zhang, J. T. Yu, Y. Yang, J. Yang, W. Zhang, and L. Tan, "Association analysis of GSK3B and MAPT polymorphisms with Alzheimer's disease in Han Chinese," Brain Research, vol. 1391, pp. 147-153, 2011.

[46] F. Massoud and G. C. Leger, "Pharmacological treatment of Alzheimer disease," Canadian Journal of Psychiatry, vol. 56, no. 10, pp. 579-588, 2011.

[47] F. Hernández, E. Langa, R. Cuadros, J. Avila, and N. Villanueva, "Regulation of GSK3 isoforms by phosphatases PP1 and PP2A," Molecular and Cellular Biochemistry, vol. 344, no. 1-2, pp. 211-215, 2010. 


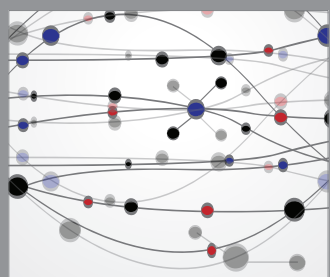

The Scientific World Journal
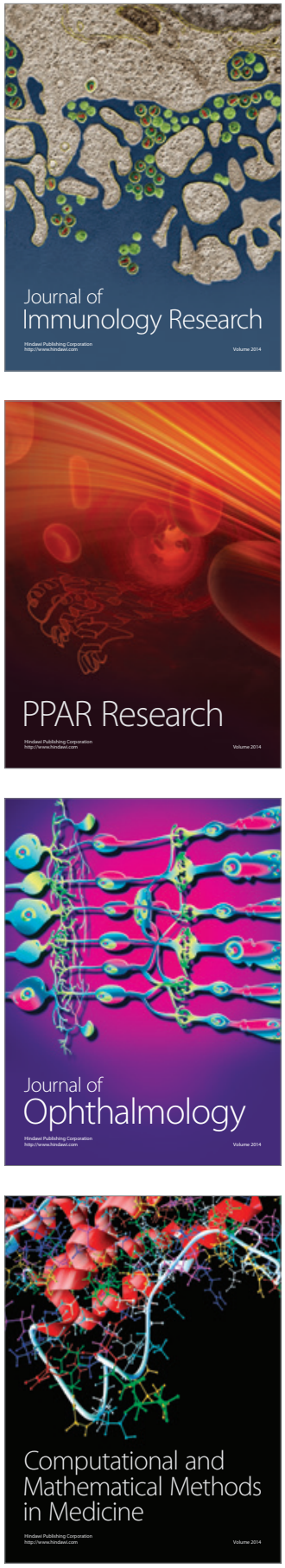

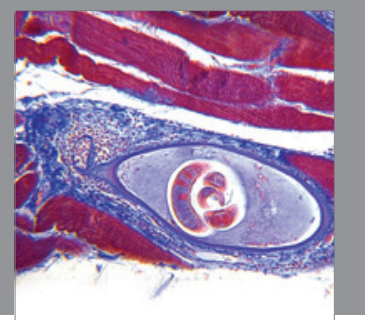

Gastroenterology

Research and Practice
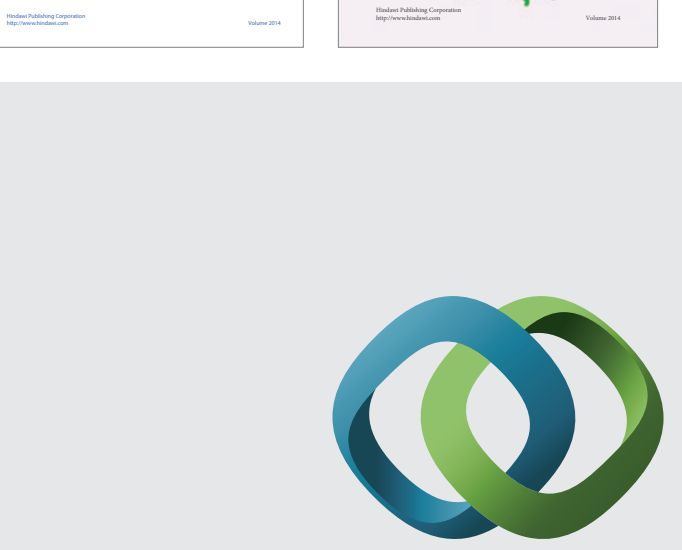

\section{Hindawi}

Submit your manuscripts at

http://www.hindawi.com
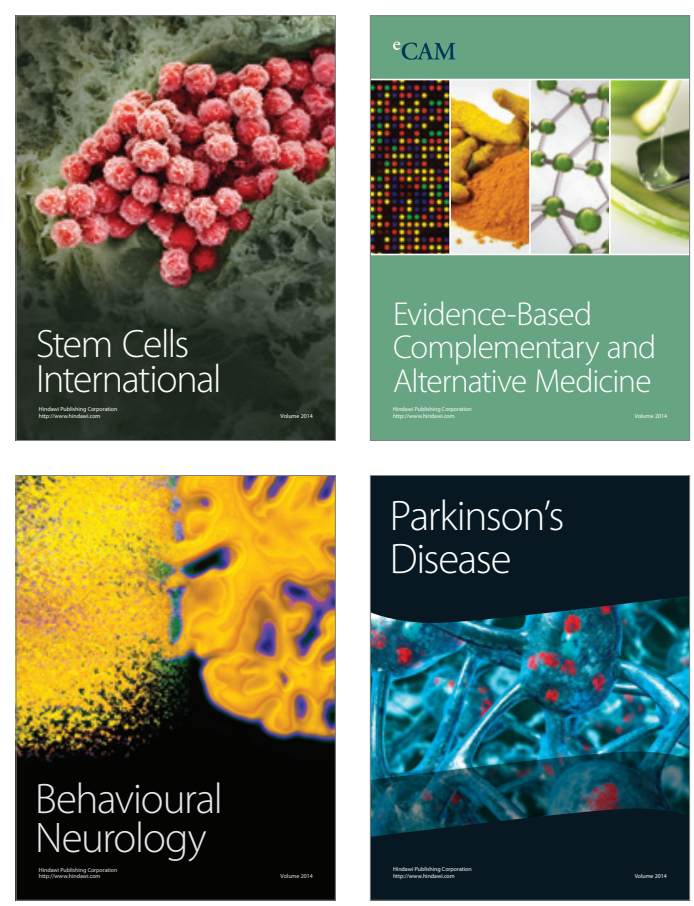

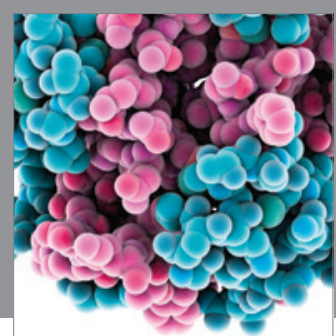

Journal of
Diabetes Research

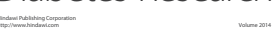

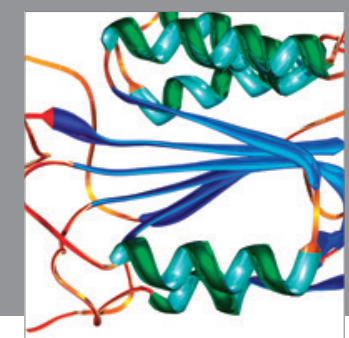

Disease Markers
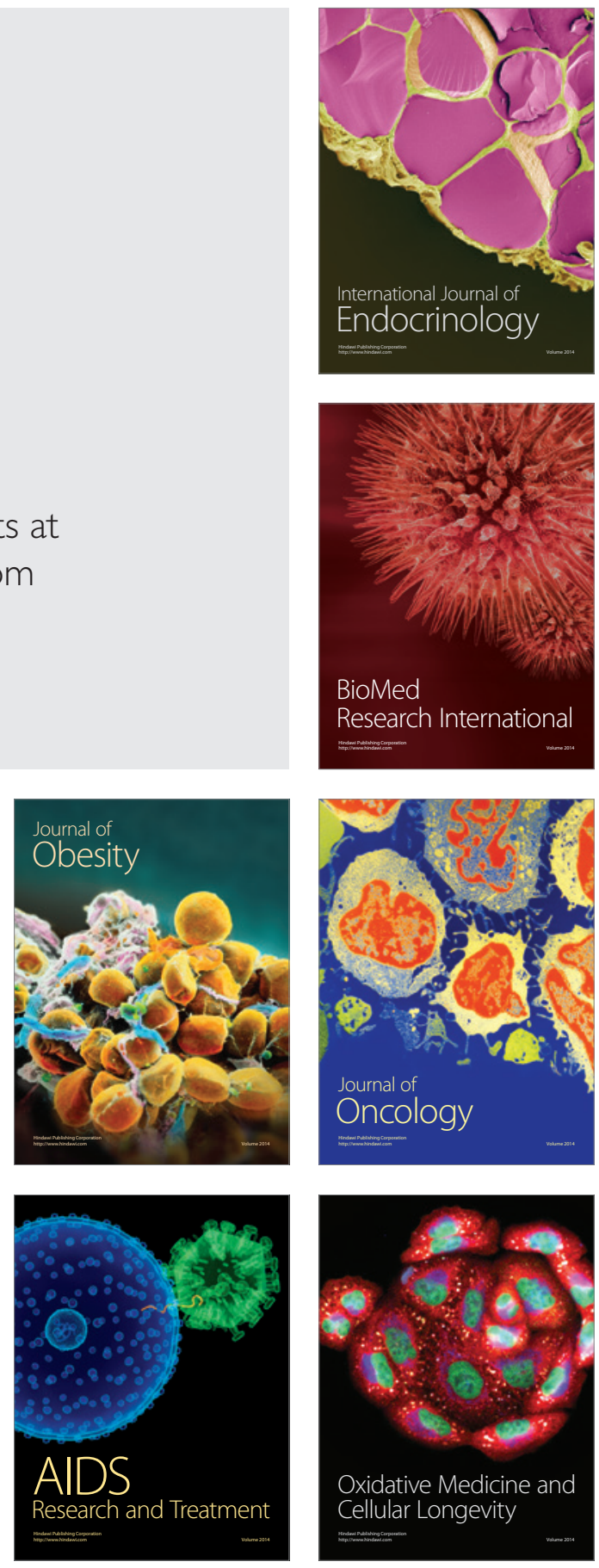